\title{
Feeling Something without Knowing Why: Measuring Emotions toward Archetypal Content
}

\author{
Huang-Ming Chang ${ }^{1,2}$, Leonid Ivonin ${ }^{1,2}$, Wei Chen ${ }^{2}$, and Matthias Rauterberg ${ }^{2}$ \\ ${ }^{1}$ CETpD Research Center, Universitat Politècnica de Catalunya \\ ${ }^{2}$ Dept. Industrial Design, Eindhoven University of Technology \\ \{H.M.Chang, L.Ivonin, W. Chen, G.W.M.Rauterberg\}@tue.nl
}

\begin{abstract}
To enhance communication among users through technology, we propose a framework that communicates 'pure experience.' This framework can be achieved by providing emotionally charged communication. To initiate this undertaking, we propose to explore materials for communicating human emotions. Research on emotion mainly focuses on emotions that are relevant to utilitarian concerns. Besides the commonly-known emotions like joy and fear, there are non-utilitarian emotions, such as aesthetic emotions, which are essential to our daily lives. Based on Jung's theory of collective unconsciousness, we consider archetypal content as a new category of affective stimuli of non-utilitarian emotions. We collected pictures and sounds of the archetype of the self, and conducted an experiment with existing affective stimuli of utilitarian emotions. The results showed that archetypal content is potential to be a new category of affective content. It is promising to explore other affective content for further studies.
\end{abstract}

Keywords: affective computing, non-utilitarian emotion, archetypal content.

\section{Introduction}

As a vision of affective computing, the new class of intelligent system is expected to be capable of capturing emotional responses from users. By analyzing these responses, this system should also be able to generate corresponding information and communicate it expressively, either back to the user herself or to another user [1]. In our previous work [2], we proposed a conceptual framework of communication among users and computers through expanding the richness of the emotional information (see figure 1.). This framework follows the paradigms in psychological experiments, which can be simply broken down to two main processes: emotion recognition and emotion elicitation [3]. In this sense, the driving question appears to be what kind of content can be used to deliver emotional information. As a starting point of this direction, we propose to follow the paradigms in psychological studies on emotion to explore affective content for communicating emotions.

Emotion has been intensively discussed in the field of psychology. However, the mainstream of psychological studies mostly focuses on explicit emotions or utilitarian emotions [4]. These types of emotions can be considered utilitarian in the sense of 
facilitating our adaptation to events that have important consequences for our wellbeing, such as anger, fear, joy, disgust, and sadness. On the other hand, there are also other types of emotions that occur without attention or intention [5]. Nonutilitarian emotions are rarely discussed but still play important roles in our daily lives, e.g. aesthetic emotion. This kind of emotions is more delicate and difficult to describe. To enrich the diversity of affective content, we attempt to explore new content for non-utilitarian emotions. In this paper, we introduce the theory of the collective unconscious proposed by psychologist Carl Jung [6]. Based on this theory, we develop a new category of affective content that are considered to be nonutilitarian. An experiment was performed to investigate how people feel about this new category of affective content. The results are discussed in the later section. Then we provide our conclusion and future work in this direction.

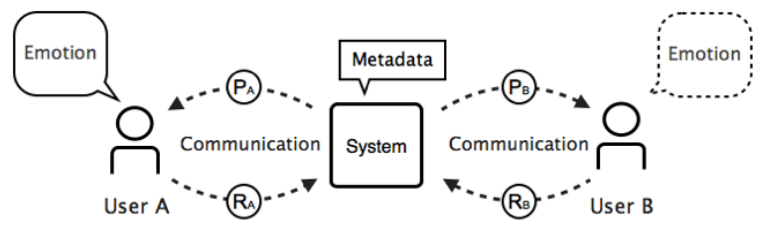

Fig. 1. An intelligent system enables emotionally charged interaction (adapted from [2]). $R_{A}$ and $\mathrm{R}_{\mathrm{B}}$ are emotion recognition; $\mathrm{P}_{\mathrm{A}}$ and $\mathrm{P}_{\mathrm{B}}$ are presenting stimuli as triggers to induce emotions in the receiver.

\section{Psychological Approach to Explore Affective Content}

There are many ways to elicit specific emotions under laboratory settings, e.g. hypnosis, imagery, and presenting affective stimuli [7]. For our purpose of exploring affective content for communicating emotions, presenting affective stimuli would probably be the most feasible and straightforward approach. The essence of this technique is to present selected audiovisual material to participants and measure their responses to these stimuli. Unlike the approaches involving confederate interaction procedures, this method may not provide psychological responses of high intensity but it ensures high degree of standardization [3]. As a benchmark for exploring new content, we look for reliable resources that provide affective stimuli with well documented results. Bradley and Lang developed the International Affective Picture System (IAPS) [8], and the International Affective Digital Sound System (IADS) [9], which are two of the broadly-used databases to investigate the correlation between subjects' self-reported emotions and the presented affective stimuli. IAPS and IADS are being developed to provide dimensional ratings of emotions for a large set of emotionally-evocative, internationally-accessible stimuli that include content across a wide range of semantic categories [8,9].

The critical part of our research question is how we can define the new category of the affective content outside of the scope of existing psychological models of emotion. Psychologist Carl Jung proposed the idea of collective unconsciousness, saying that in contrast to the personal psyche, the unconsciousness has some content 
and modes of behavior that are identical in all human beings [6]. The collective unconsciousness constitutes a common psychic substrate of a universal nature which is present in every human being. Jung further argued that the collective unconsciousness contains archetypes: ancient motifs and predispositions to patterns of behavior that manifest symbolically as archetypal images in dreams, art or other cultural forms [10]. Jung's theory was of great interests to some scholars. They therefore built an database - the Archive for Research in Archetypal Symbolism (ARAS) [11], which is a pictorial and written archive of mythological, ritualistic, and symbolic pictures from all over the world and from all epochs of human history.

Mandala, a cultural symbol originated in India, was considered as a typical archetypal symbol of Self [6]. The very basic form of Mandala is composed of one circle with one dot in its center, which shows the same pattern with the Celtic cross, the halo, the aureole, and rose windows (see Figure 2). Contemporary psychotherapists use Mandala drawing as a basic tool for self-awareness, selfexpression, conflict resolution, and healing [12,13]. Furthermore, it could also be an assessment tool for patients to communicate their physical condition in a non-verbal manner [14]. It seems that the pictures of Mandala are potential to be the new category of affective content especially Mandala does not contain any utilitarian concerns due to the fact that the content of it is merely pure symmetric pattern in a circle.

(a)



(b)

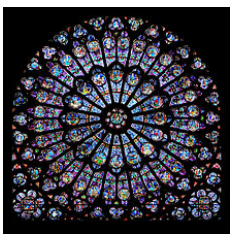

Fig. 2. (a) traditional Tibetan Mandala (book cover of [10]) and (b) the Rose Window in western cultures (the window of the north rose of Notre Dame church, Paris [15])

As for archetypal sounds, very few resources can be found. 'Om' [16] and Solfeggio Frequencies [17] would probably the only resource can be considered as archetypal sounds. 'Om' or 'Aum' is a sacred syllable in Indian religions [16]. 'Om' is the reflection of the absolute reality without beginning or the end and embracing all that exists [18]. Moreover, Solfeggio frequencies are a set of six tones that were used centuries ago in Gregorian chants and Indian Sanskrit chants (i.e. 'Om'.) These chants contained special tones that were believed to impart spiritual blessings during religious ceremonies [17]. Solfeggio frequencies introduce the common fundamental sound that is both used in western Christianity and eastern Indian religions, which strongly resonate on Jung's theory of archetypal symbols. Therefore we included them as archetypal sounds in our study.

In order to perform our experiment in a limited period of time, we needed to categorize the existing stimuli and select proper number of stimuli for each category. We followed a previous study [19], which classified the stimuli in IAPS into four categories: Positive-Arousing (PA), Positive-Relaxing (PR), Neutral (NT), and 
Negative (NG). These four categories serve as the benchmark of stimuli of utilitarian emotions for comparison. We added Mandala pictures and Om sounds as the fifth category in our study.

\section{Experiment}

The experiment followed within-subjects design. Therefore each session only accommodated one participant, and each participant viewed all the stimuli in a random order. At the beginning each participant was asked to sit in front of a monitor for displaying visual stimuli and two speakers for playing audio stimuli. The experiment was built with web-based system. All the experimental data were stored online in the database for further analysis. Before the real experiment started, each participant went through a tutorial to get familiar with the controls and the interface. After the introduction, two sessions started one after the other: picture session and sound session. During each session, the screen or the speakers presented one stimulus at a time for six seconds in a random order. After presenting each stimulus, the interface would pause for five seconds and then show the self-report screen to the participant. Our study followed the method used by IAPS [8] and IADS [9], utilizing the Self-Assessment Manikin scales (SAM) [20] for reporting their current emotional feelings, which consisted of three dimensions: valence, arousal, and dominance. No time limit for the participant to report his or her emotional feelings. Another 5-second pause appeared after the self-report, which was meant to let participants calm down and recover from the previously induced emotion. Then the next picture or sound clip was shown or played. All of the participants ran through the whole procedure individually.

We recruited 37 healthy participants, including 17 males (Mean age=, 26.00, Std. Deviation $=4.062$ ) and 20 females (Mean age=, 27.35, Std. Deviation =7.322). Most of the participants are students and researchers associated with Eindhoven University of Technology. The participants had diverse nationalities: 17 from Asia (China, India, Indonesia, and Taiwan), nine from Europe (Belgium, the Netherlands, Russia, Spain, and Ukraine), eight from Middle East (Turkey and United Arab Emirates), and three from South America (Colombia and Mexico).

IAPS and IADS contain huge amounts of visual and audio stimuli, including 1194 pictures and 167 sound clips. However, we needed to select a reasonable number of stimuli for conducting a within-subject experiment. Therefore we decided to pick six stimuli for each category to control the duration of each session within one hour. The selection of the stimuli took into account not only the embedded emotional value provided by IAPS and IADS but also the content of the stimuli. This is to ensure the diversity of the stimuli that would enhance the validity of our experiment. For example, the most positive and arousing content (which is PA category) are usually relevant to erotic pictures or sounds. If we had solely selected erotic content as PA category, the validity of this category would have become questionable. We have to include other featured content such as adventure, sports, and delicious food. Although we had tried to pick stimuli in a reasonable manner, the selection might still be biased because the selection work relied on our own judgment. 
The same criteria were used to select the materials for the fifth category archetypal content (AR), with the main difference that the distribution of archetypal content in the affective space is not defined yet. To sum up, there were two kinds of media, which were pictures and sound clips; each media type contained five categories, which were mentioned above as PR, PA, NT, NG, and AR; each category comprised of 6 stimuli. In total, 30 pictures and 30 sound clips were selected and included as experimental materials in our experiment (see table 1).

Table 1. An overview of the stimuli used in our experiment. Each medium consists of five categories: Positive-Arousing (PA), Positive-Relaxing (PR), Neutral (NT), Negative (NG), and Archetypal (AR).

\begin{tabular}{clll}
\hline Media & Category & Stimuli (Code Number or Name) & Source \\
\hline Picture & PA & $4652,4668,7405,8080,8179,8490$ & IAPS \\
\cline { 2 - 4 } & PR & $1605,1610,2060,2260,5760,5891$ & IAPS \\
\cline { 2 - 4 } & NT & $5530,7000,7050,7090,7175,7705$ & IAPS \\
\cline { 2 - 4 } & NG & $3053,3170,6230,6350,9321,9412$ & IAPS \\
\cline { 2 - 4 } & AR & $\begin{array}{l}\text { 3Hc.041, 3Pa.208, 5Ef.007, 7Ao.014, Mandala001 [10], } \\
\text { the Wheel of Life [21] }\end{array}$ & ARAS[11] \\
\hline Sound & PA & $110,201,215,352,360,367$ & IADS \\
\cline { 2 - 4 } & PR & $150,151,172,230,726,810$ & IADS \\
\cline { 2 - 4 } & NT & $171,262,376,377,708,720$ & IADS \\
\cline { 2 - 4 } & NG & $115,255,260,277,286,424$ & IADS \\
\cline { 2 - 4 } & AR & SF396Hz, SF417Hz, SF528Hz, SF639 Hz, SF741 Hz, Om & [22] \\
\hline
\end{tabular}

\section{$4 \quad$ Results and Discussion}

An appropriate statistical test for the design of our experiment would be multivariate analysis of variance (MANOVA) for repeated measures. It showed significant main effects on the variable of 'media type' $(\mathrm{F}(3,34)=3.596, \mathrm{p}=0.023$, Wilks' Lambda) and 'category' $(\mathrm{F}(12,375.988)=67.870, \mathrm{p}<0.001$, Wilks' Lambda). There also exists significance on the interaction between 'media type' and 'category' (F (12, 375.988) $=4.629, \mathrm{p}<0.001$, Wilks' Lambda). Next, we proceeded to look into the test of (univariate) repeated measures ANOVA (Huynh-Feldt) on the variable 'category', the three affective ratings all show significance: valence $(\mathrm{F}(3.181,114.514)=257.641$, $\mathrm{p}<0.001)$, arousal $(\mathrm{F}(3.321,119.546)=81.302, \mathrm{p}<0.001)$, dominance $(\mathrm{F}(2.414$, $86.898)=28.025, \mathrm{p}<0.001)$.

We performed the tests of within-subject contrasts on affective ratings to see if the emotions induced by archetypal category are different from other four categories of utilitarian emotions (see table 2). Archetypal content was set as the reference category to be compared. The tests on valence dimension between archetypal content (including both pictures and sounds) and other four categories all show significance. Then we look into the descriptive statistics. For both media (pictures and sounds), the 
rating of archetypal content on valence was lower than 'positive relaxing' and 'positive arousing' categories, higher than 'neutral' and 'negative' categories. The explained variance of 'positive relaxing' $\left(\boldsymbol{\eta}^{2}=0.780\right)$ and 'negative' $\left(\boldsymbol{\eta}^{2}=0.912\right)$ are remarkably high, whereas 'positive arousing' $\left(\boldsymbol{\eta}^{2}=0.275\right)$ and 'neutral' $\left(\boldsymbol{\eta}^{2}=0.103\right)$ are relatively low. Same tests were performed on arousal and dominance dimensions. Along these two dimensions, the results only show significance among 'positive arousing' and 'negative' categories. Then we looked into the descriptive statistics (see table 2). For both media (pictures and sounds), the arousal rating of archetypal category is lower than 'positive arousing' and 'negative' categories; the dominance rating of archetypal category is lower than 'positive arousing' category, but higher than 'negative' category.

Table 2. Statistical results of the affective ratings (valence, arousal, and dominance) on each category of pictures and sounds. Specification of effect column shows the results of the tests of within-subject contrasts on affective ratings, comparing archetypal category (AR) with each of the four categories (PR, PA, NT, and NG).

\begin{tabular}{|c|c|c|c|c|c|c|c|c|}
\hline \multirow{2}{*}{$\begin{array}{l}\text { Rati } \\
\text { ng }\end{array}$} & \multirow{2}{*}{$\begin{array}{l}\text { Cate } \\
\text { gory }\end{array}$} & \multicolumn{2}{|c|}{ Picture } & \multicolumn{2}{|l|}{ Sound } & \multicolumn{3}{|c|}{ Specification of effect } \\
\hline & & Mean & Std.Er & Mean & Std.Er & F value & $\mathbf{P}$ & $\eta^{2}$ \\
\hline \multirow{5}{*}{$\begin{array}{l}\text { Val } \\
\text { ence }\end{array}$} & $\mathrm{AR}$ & 0.914 & 0.154 & 0.446 & 0.167 & - & - & - \\
\hline & PR & 2.279 & 0.120 & 1.743 & 0.127 & $F(1,36)=127.905$ & $<0.001 * * *$ & 0.780 \\
\hline & PA & 1.509 & 0.162 & 1.293 & 0.159 & $\mathrm{~F}(1,36)=13.629$ & $0.001 * * *$ & 0.275 \\
\hline & NT & 0.631 & 0.122 & 0.248 & 0.102 & $\mathrm{~F}(1,36)=4.151$ & $0.049 *$ & 0.103 \\
\hline & NG & -2.766 & 0.151 & -2.243 & 0.119 & $\mathrm{~F}(1,36)=373.370$ & $<0.001 * * *$ & 0.912 \\
\hline \multirow{5}{*}{$\begin{array}{l}\text { Aro } \\
\text { usal }\end{array}$} & $\mathrm{AR}$ & -0.527 & 0.193 & -0.586 & 0.196 & - & - & - \\
\hline & PR & -1.095 & 0.242 & -0.608 & 0.174 & $\mathrm{~F}(1,36)=1.962$ & 0.170 & 0.052 \\
\hline & PA & 1.261 & 0.180 & 1.189 & 0.120 & $\mathrm{~F}(1,36)=130.282$ & $<0.001 * * *$ & 0.784 \\
\hline & NT & -0.833 & 0.188 & -0.203 & 0.131 & $\mathrm{~F}(1,36)=0.082$ & 0.776 & 0.002 \\
\hline & NG & 1.649 & 0.214 & 1.694 & 0.155 & $\mathrm{~F}(1,36)=188.628$ & $<0.001 * * *$ & 0.840 \\
\hline \multirow{5}{*}{$\begin{array}{l}\text { Do } \\
\text { min } \\
\text { ance }\end{array}$} & $\mathrm{AR}$ & 0.324 & 0.148 & 0.432 & 0.185 & - & - & - \\
\hline & PR & 0.617 & 0.216 & 0.604 & 0.151 & $F(1,36)=2.296$ & 0.138 & 0.060 \\
\hline & PA & 0.901 & 0.182 & 0.730 & 0.145 & $F(1,36)=6.191$ & $0.018 *$ & 0.147 \\
\hline & NT & 0.423 & 0.143 & 0.009 & 0.090 & $\mathrm{~F}(1,36)=2.929$ & 0.096 & 0.075 \\
\hline & NG & -1.243 & 0.281 & -1.054 & 0.205 & $F(1,36)=36.969$ & $<0.001 * * *$ & 0.507 \\
\hline
\end{tabular}

(* means $\mathrm{p}$ value $<0.05$, which shows significance; $* *$ means $\mathrm{p}$ value $<0.01$, which shows high significance; $* * *$ means $\mathrm{p}$ value $<=0.001$, which shows very high significance.)

Scatterplots of the ratings on valence and arousal (see Figure 3) provides a general overview of archetypal content and plot the other four categories in affective space. It needs to be noticed that the distribution of the 'archetypal' category (both pictures and sounds) in the affective space is very close to the 'neutral' category. Moreover, the significance appears to be very weak $(p=0.049)$ and the explained variance is 
relatively low $\left(\boldsymbol{\eta}^{2}=0.103\right)$. Therefore, we performed an in-depth analysis specifically on the comparison between the 'archetypal' category and the 'neutral' category. It still showed significant main effects on the variable of 'media type' $(F(3,34)=4.218$, $\mathrm{p}=0.012$, Wilks' Lambda) and the interaction between 'media type' and 'category' $(\mathrm{F}(3,34)=6.162, \mathrm{p}=0.002$, Wilks' Lambda). However, the significance on the variable 'category' disappeared $(\mathrm{F}(3,34)=1.946, \mathrm{p}=0.141$, Wilks' Lambda). In order to have a clear view of the difference between the archetypal content and the neutral content, we proceeded to conduct the same test on different media type (pictures and sounds) separately. The results can be found in table 3 .
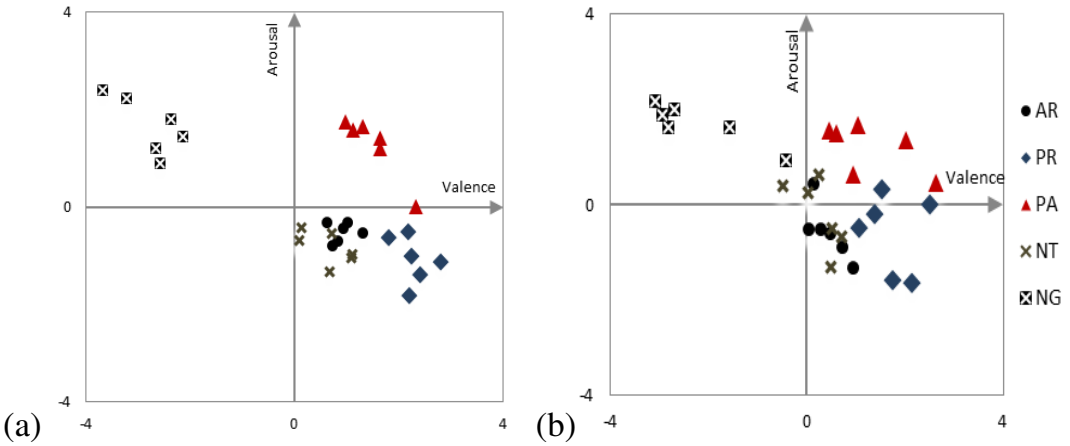

Fig. 3. Scatterplots for participants' rating of valence and arousal on affective pictures (a) and sounds (b). Archetypal (AR), Positive-Relaxing (PR), Positive-Arousing (PA), Neutral (NT), Negative (NG).

Table 3. Statistical results of the affective ratings (valence, arousal, and dominance) for the Archetypal (AR) and the Neutral (NT) category on different media types

\begin{tabular}{llll}
\hline Media & Valence & Arousal & Dominance \\
\hline Picture & $\mathrm{F}(1,36)=5.151$ & $\mathrm{~F}(1,36)=5.820$ & $\mathrm{~F}(1,36)=0.911$ \\
& $\mathrm{p}=0.029 *$ & $\mathrm{p}=0.021 *$ & $\mathrm{p}=0.346$ \\
Sound & $\mathrm{F}(1,36)=1.321$ & $\mathrm{~F}(1,36)=2.764$ & $\mathrm{~F}(1,36)=7.040$ \\
& $\mathrm{p}=0.258$ & $\mathrm{p}=1.05$ & $\mathrm{p}=0.012 *$ \\
\hline
\end{tabular}

(* means $\mathrm{p}$ value $<0.05$, which shows significance)

For the media type 'picture', it demonstrated significant effects on the valence dimension $(\mathrm{F}(1,36)=5.151, \mathrm{p}=0.029)$ and the arousal dimension $(\mathrm{F}(1,36)=5.820, \mathrm{p}=$ 0.021 ). For the other media type 'sound', only the dominance value shows significance $(F(1,36)=7.040, p=0.012)$. Bringing all the above analysis together, it can be argued that the emotions induced by archetypal content are distinctive from most of the utilitarian emotions. Although the differences between archetypal content and the neutral content are relatively minor, they can still be differentiated if we compare them only with the same media type.

Since our main purpose is to enable an intelligent system to recognize human emotions, we need to build and evaluate predictive models. Because we have already known the previous tests have shown significant main effects on the media type, we 
therefore fed the SAM ratings for affective pictures and sounds separately into Linear Discriminant Analysis (LDA), and obtained two predictive models. We present the confusion matrices generated by LDA to evaluate how well the model can predict stimuli of each category based on the data of the SAM scale. Only the cross-validated results are reported. The predictive model derived from LDA on the data for affective pictures obtains $55.8 \%$ accuracy and the effect size is large (canonical correlation = 0.770). On the other hand, the predictive model derived from LDA on the data for affective sounds obtains an accuracy of $49.4 \%$ and the effect size is also large (canonical correlation $=0.679)$. Based on the obtained confusion matrices (see table 4 and table 5), we can see that all the four stimuli of utilitarian emotions can be easily recognized, which means that the selected stimuli are nicely chosen to be a benchmark. However, archetypal pictures are more likely to be recognized as the neutral or positive relaxing pictures. Meanwhile, archetypal sounds can be correctly recognized up to $31.1 \%$ accuracy. To summarize, although archetypal pictures and sounds are significantly different from other four categories of stimuli of utilitarian emotions, the predictive models seem still not robust enough for emotion recognition.

Table 4. The confusion matrix of the model obtained from LDA on the SAM ratings for the affective pictures [count (percentage)]. Archetypal (AR), Positive-Relaxing (PR) PositiveArousing (PA), Neutral (NT), Negative (NG). Canonical Correlation $=0.770$, Effect Size $=$ Large, $55.8 \%$ of the cross-validated grouped cases are correctly classified. The cell with bold numbers means the percentage where the category was correctly predicted.

\begin{tabular}{lllllll}
\hline \multirow{2}{*}{ Category } & \multicolumn{2}{l}{ Predicted Group Membership } & \multicolumn{2}{c}{ Total } \\
\cline { 2 - 6 } & AR & PR & PA & NT & NG & \\
\hline AR & $\mathbf{2 7 ( 1 2 . 2 \% )}$ & $54(24.3 \%)$ & $28(12.6 \%)$ & $105(47.3 \%)$ & $8(3.6 \%)$ & $222(100 \%)$ \\
\hline PR & $15(6.8 \%)$ & $\mathbf{1 2 3}(\mathbf{5 5 . 4 \% )}$ & $52(23.4)$ & $27(12.2 \%)$ & $5(2.3 \%)$ & $222(100 \%)$ \\
\hline PA & $15(6.8 \%)$ & $32(14.4 \%)$ & $\mathbf{1 3 7 ( 6 1 . 7 \% )}$ & $15(6.7 \%)$ & $23(10.4 \%)$ & $222(100 \%)$ \\
\hline NT & $21(9.5 \%)$ & $35(15.8 \%)$ & $20(9.0 \%)$ & $\mathbf{1 3 8}(\mathbf{6 2 . 2 \%})$ & $8(3.6 \%)$ & $222(100 \%)$ \\
\hline NG & $5(2.3 \%)$ & $2(0.9 \%)$ & $6(2.7 \%)$ & $15(6.8 \%)$ & $\mathbf{1 9 4 ( 8 7 . 4 \% )}$ & $222(100 \%)$ \\
\hline
\end{tabular}

Table 5. The confusion matrix of the model obtained from LDA on the SAM ratings for the affective sounds [count (percentage)]. Archetypal (AR), Positive-Relaxing (PR) PositiveArousing (PA), Neutral (NT), Negative (NG). Canonical Correlation $=0.679$, Effect Size $=$ Large, $49.4 \%$ of the cross-validated grouped cases are correctly classified.

\begin{tabular}{lllllll}
\hline \multirow{2}{*}{ Category } & \multicolumn{2}{l}{ Predicted Group Membership } & \multicolumn{2}{c}{ Total } & \\
\cline { 2 - 6 } & AR & PR & PA & NT & NG & \\
\hline AR & $\mathbf{6 9 ( 3 1 . 1 \% )}$ & $49(22.1 \%)$ & $21(9.5 \%)$ & $45(20.3 \%)$ & $38(17.1 \%)$ & $222(100 \%)$ \\
\hline PR & $29(13.1 \%)$ & $\mathbf{1 0 2}(\mathbf{4 5 . 9 \%})$ & $53(23.9)$ & $31(14.0 \%)$ & $7(3.2 \%)$ & $222(100 \%)$ \\
\hline PA & $7(3.2 \%)$ & $37(16.7 \%)$ & $\mathbf{1 2 3}(\mathbf{5 5 . 4 \% )}$ & $29(13.1 \%)$ & $26(11.7 \%)$ & $222(100 \%)$ \\
\hline NT & $64(28.8 \%)$ & $28(12.6 \%)$ & $31(14.0 \%)$ & $\mathbf{6 7 ( 3 0 . 2 \% )}$ & $32(14.4 \%)$ & $222(100 \%)$ \\
\hline NG & $3(1.4 \%)$ & $5(2.3 \%)$ & $17(7.7 \%)$ & $10(4.5 \%)$ & $\mathbf{1 8 7 ( 8 4 . 2 \% )}$ & $222(100 \%)$ \\
\hline
\end{tabular}


According to the results of our study, it is clear that archetypal stimuli (AR) are distinctive from most of the stimuli of utilitarian emotions (e.g. PA, PR, and NG). Although we can still differentiate AR and NT, it appears that the emotional responses to these two categories seemed to be very similar to each other. Nevertheless, we do not consider it as a minor finding. Instead, this can lead to many interesting discussions. First, since the SAM scale was designed focusing on the investigation of utilitarian emotions, it is unclear if it is capable of differentiating nonutilitarian emotions. Moreover, IAPS contains some stimuli of 'abstract art' that are also considered to be neutral (e.g. No.7192 in IAPS). In this sense, it seems that we need more dimensions in affective space for representing non-utilitarian emotions. Second, Jung claims that archetypes are hidden in the collective unconsciousness and cannot be accessed consciously. Although Mandala and $\mathrm{Om}$ are embodied the archetype of self as visual and audio stimuli, it is probably difficult for subjects to consciously introspect their emotions toward archetypal symbols. Regarding this issue, some previous study has suggested using physiological measurement for recognizing implicit emotions [24]. It seems promising to apply this approach for future studies in this direction.

\section{Conclusion}

Our study aims at exploring new affective content to enhance the richness of emotional information that can be used for human-computer interaction. Our preliminary findings demonstrated that archetypal symbolism could be a new resource for developing new affective content for designing emotionally charged communication. Besides the archetype of the 'self', many other kinds of archetypal content is still available, e.g. hero and shadow. Emotions that induced by these contents are still unknown. It would be a promising direction to investigate the emotional qualities induced by archetypal content, and utilize the findings to design a better media to communicate 'pure experiences' for mental well-being.

Acknowledgement. This work was supported in part by the Erasmus Mundus Joint Doctorate in Interactive and Cognitive Environments, which is funded by the EACEA Agency of the European Commission under EMJD ICE FPA n 2010-0012.

\section{References}

1. Scheirer, J., Picard, R.W.: Affective Objects. MIT Media Laboratory Perceptual Computing Section Technical Report No. 524 (1999)

2. Chang, H.-M., Ivonin, L., Chen, W., Rauterberg, M.: Lifelogging for hidden minds: Interacting unconsciously. In: Anacleto, J.C., Fels, S., Graham, N., Kapralos, B., Saif ElNasr, M., Stanley, K. (eds.) ICEC 2011. LNCS, vol.6972, pp. 411-414. Springer, Heidelberg (2011)

3. Rottenberg, J., Ray, R.D., Gross, J.J.: Emotion elicitation using films. In: Coan, J.A., Allen, J.J.B. (eds.) Handbook of Emotion Elicitation and Assessment, pp. 9-28. Oxford University Press, USA (2007) 
4. Scherer, K.R.: What are emotions? And how can they be measured? Social Science Information 44, 695-729 (2005)

5. Bargh, J.A., Morsella, E.: The unconscious mind. Perspectives on Psychological Science 3, 73-79 (2008)

6. Jung, C.G.: The Archetypes and the Collective Unconscious. Princeton University Press, Princeton (1981)

7. Gross, J.J., Levenson, R.W.: Emotion elicitation using films. Cognition and Emotion 9, 87-108 (1995)

8. Lang, P.J., Bradley, M.M., Cuthbert, B.N.: International affective picture system (IAPS): Affective ratings of pictures and instruction manual. Technical Report A-8. pp. 1-12. University of Florida, Gainesville, FL (2008)

9. Bradley, M.M., Lang, P.J.: The international affective digitized sounds (IADS-2): Affective ratings of sounds and instruction manual. Technical Report B-3. pp. 29-46. Oxford University Press, USA, Gainesville, FL (2007)

10. Jung, C.G.: Man and His Symbols. Doubleday, Garden City (1964)

11. Gronning, T., Sohl, P., Singer, T.: ARAS: Archetypal Symbolism and Images. Visual Resources 23, 245-267 (2007)

12. Kim, S., Kang, H.S., Kim, Y.H.: A computer system for art therapy assessment of elements in structured mandala. The Arts in Psychotherapy 36, 19-28 (2009)

13. Schrade, C., Tronsky, L., Kaiser, D.H.: Physiological effects of mandala making in adults with intellectual disability. The Arts in Psychotherapy 38, 109-113 (2011)

14. Elkis-Abuhoff, D., Gaydos, M., Goldblatt, R., Chen, M., Rose, S.: Mandala drawings as an assessment tool for women with breast cancer. The Arts in Psychotherapy 36, 231-238 (2009)

15. Wikipedia: Rose window - Wikipedia, the free encyclopedia, http: //en.wikipedia.org/w/index.php?title= Rose_window\&oldid=510855813

16. Wikipedia contributors: Om, http://en.wikipedia.org/wiki/Om

17. Wikipedia contributors: Solfeggio frequencies, http://en.wikipedia.org/wiki/Solfeggio_frequencies

18. Maheshwarananda, P.S.S.: The hidden power in humans: Chakras and Kundalin. Ibera Verlag (2004)

19. Ribeiro, R.L., Teixeira-Silva, F., Pompéia, S., Bueno, O.F.A.: IAPS includes photographs that elicit low-arousal physiological responses in healthy volunteers. Physiology \& Behavior 91, 671-675 (2007)

20. Bradley, M.M., Lang, P.J.: Measuring emotion: The self-assessment manikin and the semantic differential. Journal of Behavior Therapy and Experimental Psychiatry 25, 49-59 (1994)

21. Bhavacakra - Wikipedia, the free encyclopedia, http://en.wikipedia.org/wiki/Wheel_of_Existence

22. Welch, D.: mountainmystic9's Channel, http: / / www . youtube.com/user/mountainmystic9

23. ONEMIND4U: Om meditation, http: / / www . youtube. com/watch?v=imWRQpY0P58

24. Bechara, A., Damasio, H., Tranel, D., Damasio, A.R.: The Iowa Gambling Task and the somatic marker hypothesis: Some questions and answers. Trends in Cognitive Sciences 9, 159-162 (2005) 